centre of our galaxy and the distances to our two nearest extragalactic neighbours, the Magellanic Clouds. The distances of clusters depend on fitting their "zero-age main sequence" in the temperature-luminosity diagram to that of the Hyades cluster which is well determined by the moving-cluster method and confirmed-as was shown by $\mathrm{Dr}$ O. J. Eggen (Mount Stromlo Observatory, Canberra)-by entirely independent measurements of the temperatures, magnitudes and trigonometric parallaxes of nearby stars resembling the Hyades in age, motions and chemical composition. The absorption of light in interstellar space immediately surrounding our solar system was shown by Dr B. Strömgren (Copenhagen University) to be almost entirely concentrated in discrete small clouds or large cloud complexes, with almost no continuous distribution of dark matter between the clouds.

Going out to distances more comparable to the size of our galaxy, Sir Richard Woolley described the determination of luminosities of pulsating variables by the Baade-Wesselink method of comparing actual changes in radius deduced from velocity variations with the relative change deduced from variations in brightness and surface temperature. In combination with the theory of pulsation, this method leads to quite low masses for many variables which in turn suggests that they could have lost mass in the course of their evolution. Dr W. Becker (Basle University) described a procedure for building up a contour map of star density in the disk and halo of our galaxy by photometry in red, green and ultraviolet bands of the spectrum which enables faint stars of different luminosity and chemical composition to be sorted out. Dr A. Toomre (Massachusetts Institute of Technology) showed that the distortion of the central plane of our galaxy could be explained by tidal action of the Magellanic Clouds provided that the Earth's distance from the centre of our galaxy does not exceed 8 kiloparsecs (compared with the round figure of 10 kiloparsecs adopted by the International Astronomical Union some years ago). But an attempt to improve galactic constants by studying proper motions was found by Dr S. V. M. Clube (Royal Greenwich Observatory) to lead to inconclusive results.

Are the redshifts of quasars of cosmological origin, indicating enormous distances, or are they an intrinsic effect due to other causes? Arguments on each side were reviewed by $\mathrm{Dr} \mathrm{M}$. Schmidt (California Institute of Technology), who showed that the hypothesis of an intrinsic origin would require a revision of present-day concepts in physics, but that the cosmological hypothesis is not fatally in conflict with any results obtained to date.

\section{X-RAY ASTRONOMY

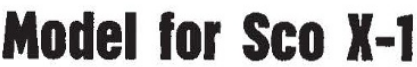

by our Cosmology Correspondent

Since the discovery that Sco $\mathrm{X}-1$ is a triple radio source with some features like those of a pulseless pulsar, there has been speculation that the source might indeed belong to the same family as pulsars (see Nature Physical Science, 229, 218; 1971). K. Davidson, F. Pacini and E. E. Salpeter have now published a detailed model for the thermal X-ray emission from Sco X-1 in terms of a hot gaseous region surrounding a rotating neutron star (Astrophys. J., 168, 45; 1971). Although this model was developed before the recent flurry of activity which has resulted, in the discovery of periodic variations in Centaurus X-3 and Cygnus X-1, these discoveries can, on the face of it, only add to the interest in models such as that of Davidson et al.

With all models of Sco X-1, the fundamental problem is the generation of thermal bremsstrahlung-the source has maintained a temperature of roughly $5 \times 10^{7} \mathrm{~K}$ for a decade, and it has been widely accepted that the basic energy source must be gravitational, with accre- tion of gas onto a white dwarf releasing energy as proposed by Prendergast and Burbidge in 1968. But the difficulty of explaining the detailed fiaring and flickering of Sco X-1 at both optical and $\mathrm{X}$-ray frequencies, together with the presence of intermittent periodic and quasi-periodic structure in the light curves from the source, makes it desirable that other models should also be studied. Davidson, Pacini and Salpeter have looked at models in which the energy source is a rotating neutron star, the inspiration for this stemming from the apparent success of the rotating neutron star model in explaining the energy generation mechanism operating in pulsars.

The model which is most successful has a dense shell of gas surrounding the central pulsar. Supported by particular and electromagnetic radiation far beyond the speed of light circle, at a distance of roughly $10^{9} \mathrm{~cm}$, this cocoon is heated to the observed temperature by the radiation it absorbs. For Sco X-1, the observations require that such a shell must be quite thick, the density in the line of sight being several $\mathrm{g} \mathrm{cm}^{-2}$ so that the pulsar frequency fields do not penetrate the cocoon. Plausible values for the efficiency of the heating mechanism and the mass of the underlying star suggest a pulsar period of $3 \mathrm{~ms}$ and a gas cloud radius of $1.5 \times 10^{9} \mathrm{~cm}$. But the surface field required is only $5 \times 10^{9} \mathrm{G}$,

\title{
Infrared Variations in Seyfert Galaxies?
}

Although measurements of the infrared emission of the Seyfert galaxy NGC4151 at $11 \mu \mathrm{m}$ do not yield any evidence of significant flux changes with time, the possibility that some flux changes have occurred cannot be ruled out. That is the principal conclusion reached by Stein and Gillett in next Monday's issue of Natuire Physical Science.

These results are of particular interest because of their importance for the radiation mechanisms at infrared wavelengths greater than about $10 \mu \mathrm{m}$. One model of Seyfert galaxies, for example, proposes that there is a central luminous source of short wavelength radiation surrounded by a cloud of particles that radiate at infrared wavelengths. This model would be untenable if there were fluctuations on a time scale of less than about a year. Variations of the order of a day would also place serious constraints on models involving non-thermal infrared sources.

Quite a large amount of evidence has been accumulated for time variations of the visible and radiofrequency flux from the nuclei of Seyfert galaxies and possible variations have also been reported at the near infrared wave- lengths of about 2 to $3 \mu \mathrm{m}$; Stein and Gillett have now extended these measurements to $11 \mu \mathrm{m}$ with photometric measurements of NGC4151 made during the two years up to June 1971.

They have found two pieces of evidence that seem to suggest variations on a time scale of less than a month. In both cases the three standard deviation upper limit of one measurement was much lower than the lower limit of the flux a few days later. But Stein and Gillett are quick to point out that deductions from this can be misleading, and they apply several statistical checks. They find, for example, that the standard deviation of the distribution of their twenty-nine measurements is only slightly greater than the average standard deviation of the individual measurements whereas, if variations had occurred, larger differences would be expected. The numbers of measurements lying within one, two and three standard deviations of the mean of the twenty-nine measurements are also close to the numbers expected if there is no variation. Similar conclusions were reached after analysis of measurements on a comparison star ( $\gamma \mathrm{U} \mathrm{Ma}$ ). 